\title{
CATALYTIC COMBUSTION OF PROPANE IN A MEMBRANE REACTOR WITH SEPARATE FEED OF REACTANTS - I. OPERATION IN ABSENCE OF TRANS-MEMBRANE PRESSURE GRADIENTS
}

\author{
GUIDO SARACCO ${ }^{\dagger}$ \\ Dipartimento di Scienza dei Materiali e Ingegneria Chimica, Politecnico di Torino, C.so Duca degli \\ Abruzzi 24, 10129 Torino, Italy \\ JAN WILLEM VELDSINK \\ Chemical Engineering Department, University of Groningen, Nijenborgh 4, 9747 AG Groningen, \\ The Netherlands \\ and
}

GEERT F. VERSTEEG and WIM P. M. VAN SWAAIJ

Chemical Engineering Department, Twente University of Technology, PO Box 217, 7500 AE Enschede, The Netherlands

(Received 14 September 1994; accepted in revised form 10 January 1995)

\begin{abstract}
A pilot plant study on propane catalytic combustion in a membrane reactor with separate reactant feeds is presented. The membrane consisted of a porous alumina tube activated by insertion into its pores of a Pt $/ \gamma-\mathrm{Al}_{2} \mathrm{O}_{3}$ catalyst. The role of reactants concentration and of the feed flow rates were studied in the transport-controlled operating regime, where a number of interesting properties of this reactor setup can be exploited (absence of reactant slip through the membrane, lower risks of thermal runaways, possibility of increasing conversion by application of a pressure difference over the membrane, etc.). Attention is here focused on operation in the absence of trans-membrane pressure gradients. The reactor fluiddynamics are investigated, too. The experimental results are in good agreement with the predictions of an isothermal model, analytically solved and based on the simplifying assumption that the reaction takes place in a limited zone inside the membrane (i.e. a surface for infinitely fast reactions).
\end{abstract}

\section{INTRODUCTION}

The research area of inorganic membrane reactors has gained increasing interest in the last decade (Tsotsis et al., 1993; Saracco and Specchia, 1994). In this context, most investigations concerned the use of permselective membranes to increase conversion of catalytically promoted equilibrium reactions (Saracco et al., 1994). This kind of application exploits the ability of membranes to separate selectively at least one of the reaction products.

In recent years a new membrane reactor concept has been developed for non-separative applications according to which a catalytically active porous membrane mediates the contact and the reaction between two reactants fed from opposite sides of it as in Fig. 1 (Sloot, 1991; Veldsink, 1993). This strategy was demonstrated to be promising for those reactions which require strict stoichiometric feed of reactants such as $\mathrm{NO}_{x}$ reduction with ammonia in the presence of oxygen (Sloot, 1991), or the Claus reaction for $\mathrm{SO}_{2}$ abatement with $\mathrm{H}_{2} \mathrm{~S}$ (Sloot et al., 1990, 1992). In fact, provided the kinetics are fast enough compared with the transport of reactants, the reaction takes place in

${ }^{\dagger}$ Author to whom correspondence should be addressed. a limited zone inside the membrane (a plane, for infinitely fast reactions), and reactants reach it in proportion to their stoichiometric coefficients. Any change in reactant concentration in the gas feeds results in a shift of the reaction zone inside the membrane without losing the above property. Any slip of reactant towards the opposite side of the membrane is prevented as well.

Recent studies on $\mathrm{CO}$ catalytic oxidation, used as a model reaction, elucidated some further properties of this reactor setup which can make it attractive for hydrocarbon catalytic combustion either for heat production or for yield increase of intermediate oxidation products (Veldsink et al., 1992; Veldsink, 1993). When the conditions for the absence of any slip of reactants are achieved, the overall attainable conversion will become almost exclusively controlled by transport phenomena, which are much less temperature sensitive than kinetics, allowing to operate with lower risks of thermal runaway and consequent catalyst sintering. Furthermore, the possibility to vary independently the flow rates, the concentrations and the pressures of the two reactant feeds gives flexibility to the system and easy controllability. Moreover, the formation of explosive mixtures is hampered by avoiding any premixing of the reactants. Finally, by applying 
a pressure difference over the membrane the products can be shifted preferentially towards the low pressure chamber allowing to:

- feed one reactant (i.e. the hydrocarbon) continuously to the membrane by closing the outlet of its feed chamber (e.g. dead end system), or to recycle it;

- increase the overall conversion, limited only by the flux of the reactant which diffuses against the pressure gradient;

- reduce the residence time of the products in the catalytic membrane resulting in a higher selectivity for e.g. partial oxidation products. These products are removed from the membrane by the convective flow preventing further oxidation.

The achievements obtained in the above quoted investigations were the incentives to perform a pilot plant study on the catalytic combustion of propane with air using a tubular porous-alumina membrane activated by insertion into its pores of a $\mathrm{Pt} / \gamma-\mathrm{Al}_{2} \mathrm{O}_{3}$ catalyst. The studied reaction was

$$
\begin{gathered}
\mathrm{C}_{3} \mathrm{H}_{8}+5 \mathrm{O}_{2} \rightarrow 3 \mathrm{CO}_{2}+4 \mathrm{H}_{2} \mathrm{O}, \\
\Delta H^{\circ}=-2221 \mathrm{~kJ} \mathrm{~mol}^{-1}
\end{gathered}
$$

In this first communication of a series, the preparation, the characterisation, the performance and the modelling of the membrane reactor are addressed, focusing on the transport-controlled regime (i.e. hightemperature regime), in which no significant slip of reactants occurs and all the above underlined properties are fulfilled. Attention is paid on reactor fluiddynamics and the operation in the absence of pressure gradients across the membrane. In the second communication of this series (Saracco et al., 1995) the effect of the application of pressure differences over the membrane will be investigated and interpreted on the grounds of a more complex modelling approach than the one here presented.

\section{SOME THEORETICAL ISSUES}

When no pressure differences are applied and kinetics are fast enough only diffusive transport of reactants controls the performance of the reactor. Consider a fast irreversible reaction $A+B \rightarrow C$, where $A$ is fed at the tube-side of a tubular membrane reactor and B is fed at the shell-side (Fig. 1).

In case the reaction rate inside the membrane is fast compared to the transport rate the reaction takes place in a limited zone inside the membrane and no significant slip of reactants occurs. The flux of reactants through the membrane is increased compared with the maximum diffusive flux occurring when no reaction takes place and the reactant concentration at the opposite membrane side is set equal to zero. In line with the Van Krevelen-Hoftijzer approximation for gas-liquid absorption accompanied by a reaction (Westerterp et al., 1983), an enhancement factor can catalytic

membrane

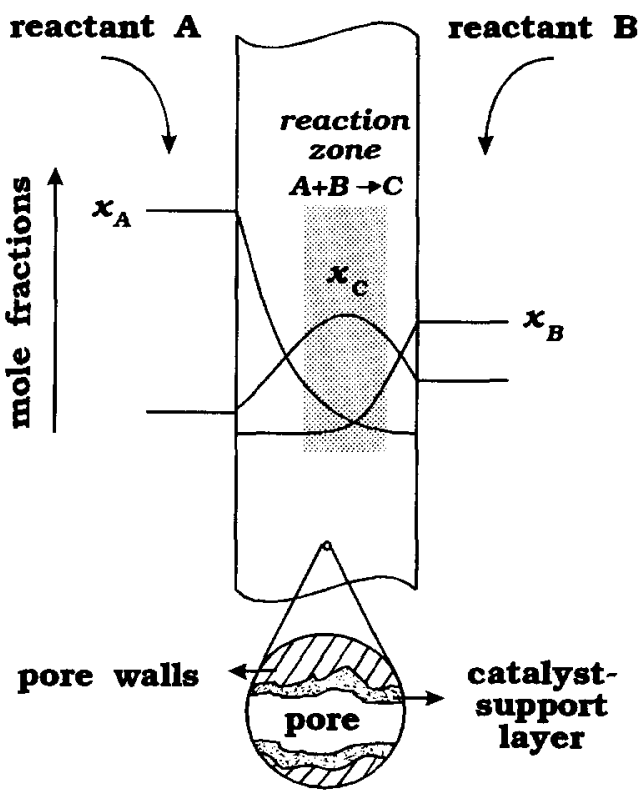

Fig. 1. Scheme of a membrane reactor with separate feed of reactants.

be defined as follows (referring to reactant A):

$$
E_{A}=\frac{r_{t} N_{A}\left(r_{t}\right)-r_{s} N_{A}\left(r_{s}\right)}{r_{t} N_{A, \mathrm{ph}}\left(r_{t}\right)}=\frac{\phi_{r A} R T}{D_{A}^{e} r_{t} p_{A}\left(r_{t}\right)} .
$$

A further parameter that can be usefully employed to characterise the reactor performance is the slip fraction, defined as follows:

$$
S_{A}=\frac{r_{s} N_{A}\left(r_{s}\right)}{r_{t} N_{A}\left(r_{t}\right)}
$$

If the reaction rate is further increased (e.g. rising the reaction temperature) the reaction zone will contract until a reaction plane is virtually obtained for infinitely fast reactions [see Fig. 2(a)]. Under these conditions the enhancement factor reaches its maximum, asymptotic value:

$$
E_{A \infty}=1+\frac{v_{A} D_{B}^{e} p_{B}\left(r_{s}\right)}{v_{B} D_{A}^{e} p_{A}\left(r_{t}\right)}
$$

and the flux of reactant $A$ becomes equal to

$$
N_{A}\left(r_{t}\right)=D_{A}^{e}\left[1+\frac{v_{A} D_{B}^{e} p_{B}\left(r_{s}\right)}{v_{B} D_{A}^{e} p_{A}\left(r_{t}\right)}\right] \frac{p_{A}\left(r_{t}\right)}{R T r_{t} \ln \left(r_{s} / r_{t}\right)} .
$$

According to the Bosanquet scheme, the effective diffusion coefficient is estimated from the Knudsenand bulk-diffusion formulations:

$$
D_{A}^{e}=\frac{1}{\frac{1}{\frac{4}{3} K_{0} \sqrt{8 R T / \pi M_{A}}}+\frac{1}{(\varepsilon / \tau) D_{A}^{0}}} .
$$

Provided the external mass transfer resistance is negligible the mole fractions $x_{A}\left(r_{t}\right)$ and $x_{B}\left(r_{s}\right)$ at the 


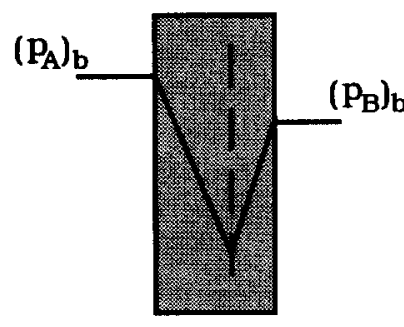

(a)

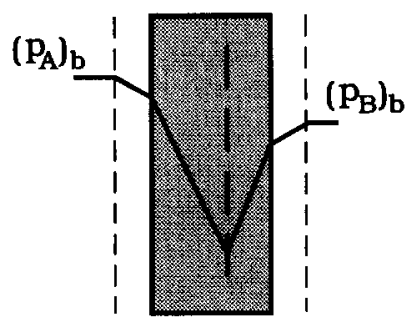

(b)

Fig. 2. Partial pressure qualitative profiles for an infinitely fast reaction with either negligible (a, model D) or not negligible (b, model $\mathbf{E})$ external mass transfer resistance.

membrane interfaces are equal to the respective bulk values $\left\langle x_{A}\right\rangle_{t}$ and $\left\langle x_{B}\right\rangle_{s}$. The modelling scheme based on this hypothesis (Dirichlet boundary conditions) will be named hereafter "model $D$ ".

If external mass transfer resistance is accounted for ["model E", Fig. 2(b)], eq. (4), referring to bulk partial pressures, becomes

$$
N_{A}\left(r_{t}\right)=\frac{D_{A}^{e}\left[1+\frac{v_{A} D_{B}^{e} p_{B}\left(r_{s}\right)}{v_{B} D_{A}^{e} p_{A}\left(r_{t}\right)}\right]}{\left[\frac{D_{A}^{e}}{r_{t}\left(k_{g A}\right)_{t}}+\frac{D_{B}^{e}}{r_{s}\left(k_{g B}\right)_{s}}+\ln \left(\frac{r_{s}}{r_{t}}\right)\right]} \frac{p_{A}\left(r_{t}\right)}{r_{t} R T} .
$$

The radius of the cylindrical reaction surface can be then easily derived: coefficients [see Reid et al. (1987)] because of its generally good performance in the operating temperature range adopted in the present investigation $\left(100-500^{\circ} \mathrm{C}\right)$ :

$$
D_{i j}^{0}=\frac{10^{-3} T^{1.75} \sqrt{\frac{M_{i}+M_{j}}{M_{i} M_{j}}}}{P\left(\sqrt[3]{v_{i}}+\sqrt[3]{v_{j}}\right)^{2}} .
$$

Equation (9) holds for binary systems. For the $\varepsilon / \tau$ calculation a reasonable approximation can be made by assuming that the two reactants $A$ and $B$ diffuse towards the reaction plane in pure inert gas (i.e. the influence of reaction products is neglected). The occurrence of temperature gradients across the mem-

$\delta_{r}=\exp \left\{\frac{\left[\frac{D_{B}^{e}}{r_{s}\left(k_{g B}\right)_{s}}+\ln r_{s}\right]-\left(E_{A \infty}-1\right)\left[\frac{D_{A}^{e}}{r_{t}\left(k_{g A}\right)_{t}}-\ln r_{t}\right]}{E_{A \infty}}\right\}$

where $E_{A \infty}$ is expressed, in line with eq. (3), as follows:

$$
E_{A \infty}=1+\frac{v_{A} D_{B}^{e} x_{B}}{v_{B} D_{A}^{e} x_{A}} .
$$

The partial pressures at the membrane interfaces or in the bulk of the reactant feeds (depending on the type of boundary conditions chosen) were assumed to be equal to the logarithmic mean between the measurable values of the partial pressures at the inlet and at the outlet of the chambers separated by the membrane. This is a consequence of assuming a plug-flow regime for the gases flowing along the membrane. In Section 7 this assumption will be discussed and demonstrated to be preferable over a CISTR approach.

If correct and accurate expressions for the estimation of the gas-phase diffusion coefficients and for the external mass transfer coefficients are adopted, $\varepsilon / \tau$ can be calculated since it is the only remaining unknown parameter in eq. (4) or eq. (6). In fact, all the other variables can be measured under reaction conditions and $K_{0}$ can be measured by permeation runs with inert gases $\left[K_{0}=7.78 \times 10^{-9} \mathrm{~m}\right.$; see Saracco et al. (1995)].

In this context, the Fuller-Schettler-Giddings equation was chosen for the estimation of diffusion brane was also assumed to be negligible. On the other hand, this equation was employed for diffusivity estimation in the flux terms of the model outlined in Table 1. Values of the diffusion volumes, $v_{i}$, are listed in Reid et al. (1987).

The external mass transfer coefficients can be evaluated as a function of the flow regime in the two opposite chambers and of their configurations. The reactant chambers in the present reactor setup were annular passages between walls having different temperatures (see Section 3). The flow rates along the membrane were always kept in the laminar regime ( $20<R e<150$ ). By considering the solution of the Graetz problem given by Lundberg et al. (1963) for annular passages and the Chilton-Colburn analogy of heat and mass transfer, the following expressions can be easily worked out for the reactor setup employed in this study:

$$
\begin{gathered}
\left(k_{g i}\right)_{t}=1.73 \frac{D_{i}^{0}}{r_{t}-r_{\text {oil }}} \\
\left(k_{g i}\right)_{s}=2.63 \frac{D_{i}^{0}}{r_{\text {shell }}-r_{s}} .
\end{gathered}
$$

These expressions are valid for fully developed flows. In fact, any entry length effect can be neglected 
Table 1. Model for a tubular membrane reactor with separate feed of reactants operated in the transport-controlled regime with no pressure difference applied over the membrane

Intra-chamber mass balances

Flow patterns: CSTR

$\frac{\phi_{A} P}{R\langle T\rangle_{A}}\left(x_{A \text { in }}-x_{\text {Aout }}\right)=-2 \pi r_{t} L N_{A}\left(r_{t}, x_{\text {Aout }}\right)$

Plug-flow $\frac{\phi_{A} P}{R\langle T\rangle_{A}} \frac{\mathrm{d}\langle x\rangle_{A}}{\mathrm{~d} z}=-2 \pi r_{t} N_{A}\left(r_{t}\right)$

Stoichiometric relationship

$N_{B}\left(r_{s}\right)=-\frac{v_{B} r_{t}}{v_{A} r_{s}} N_{A}\left(r_{t}\right)$

Intra-membrane mass balance

$$
N_{A}\left(r_{t}\right)=\frac{D_{A}^{e}\left[1+\frac{v_{A} D_{B}^{e} p_{B}\left(r_{s}\right)}{v_{B} D_{A}^{e} p_{A}\left(r_{t}\right)}\right]}{\left[\frac{D_{A}^{e}}{r_{t}\left(k_{g A}\right)_{t}}+\frac{D_{B}^{e}}{r_{s}\left(k_{g B}\right)_{s}}+\ln \left(\frac{r_{s}}{r_{t}}\right)\right]} \frac{p_{A}\left(r_{t}\right)}{r_{t} R T_{m}}
$$

in the reactor setup described below (the membrane section is sufficiently far away from all the chamber entries). Veldsink (1993) demonstrated that natural convection does not affect in a significant way the flow patterns when operating the module at the upper range of Reynolds numbers.

Based on the above hypotheses an overall set of equations describing the reactor performance was derived and listed in Table 1 for a model $E$ approach. Model $D$ equations can be attained by setting infinite values of the mass transfer coefficients. The integration of the set of equations reported in Table 1 along the membrane, assuming for both chambers a plugflow pattern, leads to the following expression of the overall conversion:

$$
\zeta_{A}=\left(1+K_{1}\right)\left[1-\exp \left(-2 \pi L K_{2}\right)\right]
$$

where

$$
\begin{array}{r}
K_{1}=\left(1-E_{A \infty}\right) \frac{1+\alpha \frac{v_{B} \phi_{A}\langle T\rangle_{A}\langle x\rangle_{A \text { in }}}{v_{A} \phi_{B}\langle T\rangle_{B}\langle x\rangle_{B \text { in }}}}{1-\alpha \frac{D_{B}^{e} \phi_{A}\langle T\rangle_{A}}{D_{A}^{e} \phi_{B}\langle T\rangle_{B}}} \\
K_{2}=\frac{\langle T\rangle_{A}}{T_{m}} \frac{\left(D_{A}^{e}-\alpha \frac{\phi_{A}\langle T\rangle_{A}}{\phi_{B}\langle T\rangle_{B}} D_{B}^{e}\right)}{\left[\frac{D_{A}^{e}}{r_{t}\left(k_{g A}\right)_{t}}+\frac{D_{B}^{e}}{r_{s}\left(k_{g B}\right)_{s}}+\ln \left(\frac{r_{s}}{r_{t}}\right)\right]}
\end{array}
$$

In the above expressions $\alpha$ has to be set equal to 1 in case the two chambers are fed countercurrently, or equal to -1 for a cocurrent feed.

On the other hand, if the two chambers are considered to be perfectly mixed (i.e. CISTR) the expression of conversion is straightforward:

$$
\zeta_{A}=\frac{2 \pi r_{t} L R\langle T\rangle_{A}}{P \phi_{A}\langle x\rangle_{A \text { in }}} N_{A}\left(\langle x\rangle_{A \text { out }}, r_{t}\right)
$$

\section{THE EXPERIMENTAL SETUP}

The experimental apparatus is schematically shown in Fig. 3, while the membrane reactor is represented in more detail in Fig. 4. The feed flow rates and compositions were controlled and dosed to each side of the membrane by means of Brooks mass flow meters. The pressures at the tube or at the shell side of the membrane were controlled via Tescom back-pressure regulators placed close to the outlet of the respective chambers, and measured via Druck pressure transducers. The operating pressure inside the reactor was generally kept equal to 2 bar. The two chambers were operated countercurrently. Thermal control was assured by a PID-regulated oven surrounding the shell of the module whose internal diameter was $50 \mathrm{~mm}$. Its function was to startup the reaction or to keep the reactor at a desired temperature level. In the tube-side of the module a stainless steel pipe (external diameter $6 \mathrm{~mm}$ ) was present, through which silicon oil was circulated at about $100^{\circ} \mathrm{C}$ for heat removal purposes. The membrane (length, $100 \mathrm{~mm}$; internal diameter, $14 \mathrm{~mm}$; external diameter, $20 \mathrm{~mm}$ ) was sealed at both ends with a multiple brazing technique to a couple of stainless steel tubes (length, $120 \mathrm{~mm}$ each) whose function was that of isolating the membrane in the central part of the reactor module where axial temperature gradients could be kept to a minimum. The sealing technique (Saracco and Specchia, 1994), delivered by Velterop BV (Twente University of Technology, Enschede), allowed operation of the reactor at temperatures as high as $800 \mathrm{~K}$, despite the thermal expansion mismatch between the two joined materials. Temperature gradients were checked with three $\mathrm{K}$ type thermocouples placed as shown in Fig. 4. The maximum temperature difference registered between the surface temperature of the membrane and the extremes of the stainless steel connection tubes was about $50^{\circ} \mathrm{C}$ when operating at very high temperatures and with high propane conversions. However, since the membrane is placed well inside the module, and, obviously, no reaction takes place on the stainless steel tubes, the temperature excursion along the membrane surface should have been in any case much lower than the above value. In fact, the temperature gradient should be likely concentrated at the membrane connections and along the stainless steel tubes, 


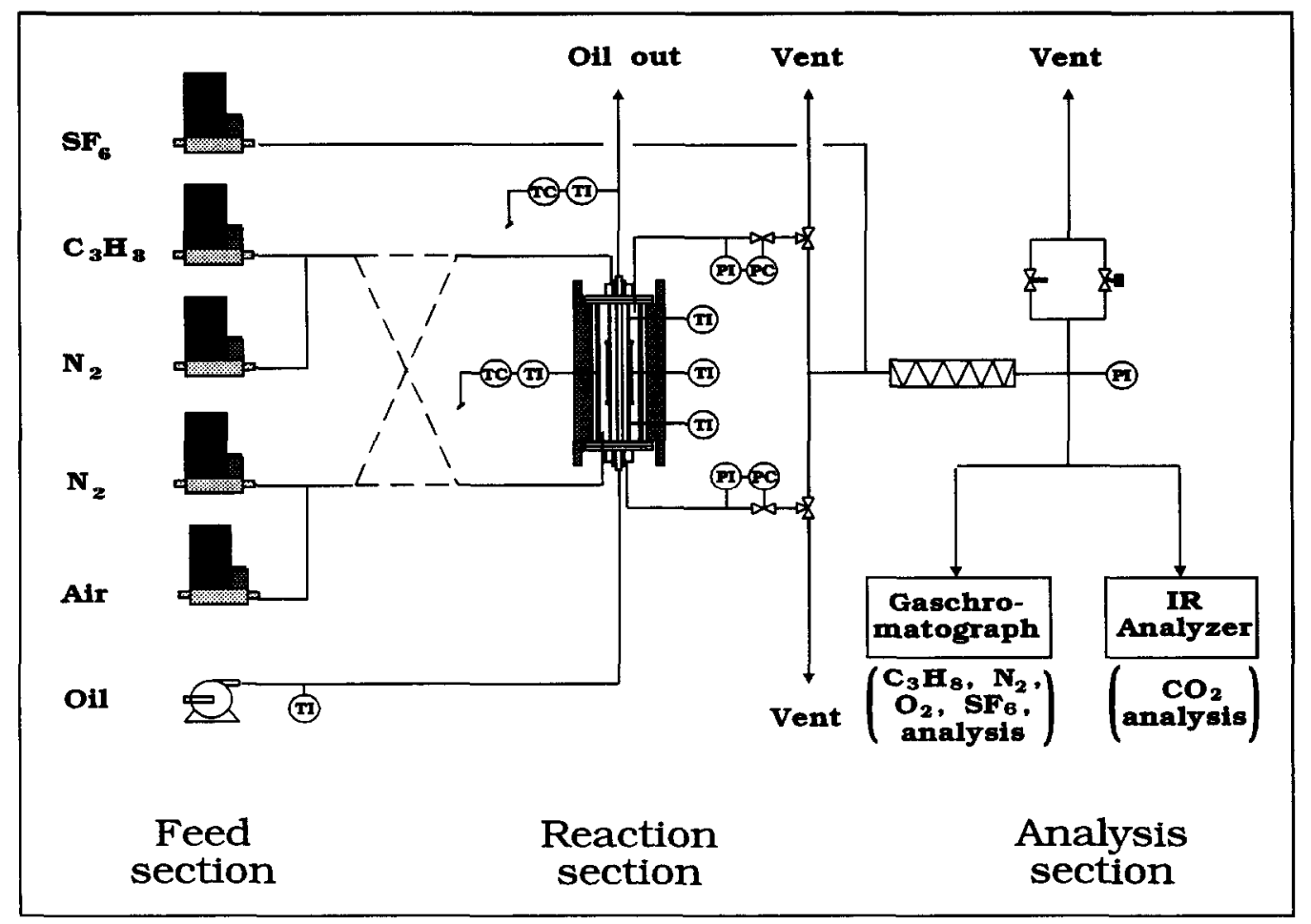

Fig. 3. Scheme of the pilot plant.

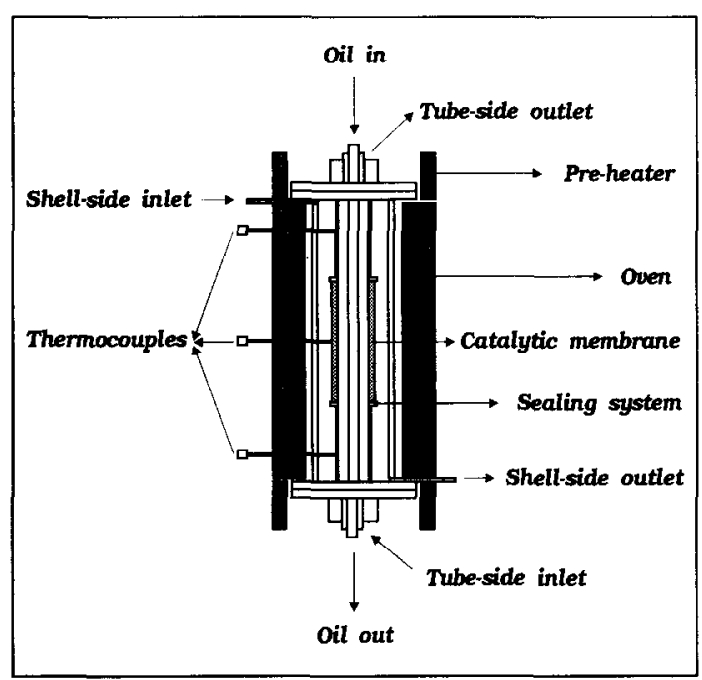

Fig. 4. Scheme of the reactor module.

acting as heat exchangers between the two gas streams.

The temperature of the shell of the module, $T_{\text {snell }}$, and of the oil flowing in the heat removal pipe, $T_{\text {oil }}$, were determined by means of specific thermocouples.

Chemical analysis of inlet and outlet flow rates was performed via a Maihak UNOR6N IR spectrometer $\left(\mathrm{CO}_{2}\right.$ analysis) and a Varian 3300 gas chromatograph equipped with a Hayesep and a MS $5 \AA$ column, placed in series, and with both a TCD and a FID detector. The use of a FID detector was necessary for a proper measurement of any slip of propane. $\mathrm{SF}_{6}$ was dosed as a tracer at a known flow rate so as to calibrate analysed effluent flow rates via gas chromatographic analysis. By these means overall mass balances of each component could be verified with deviations always less than $15 \%$.

\section{MEMBRANE PREPARATION}

The basic support consisted of a porous $\alpha$-alumina tube (nominal pore diameter, $0.7 \mu \mathrm{m}$ ). Sealings had to be already present before any activation of the membrane since the sealing procedure implies very high temperatures $\left(>1000^{\circ} \mathrm{C}\right)$, which would have promoted irreversible catalyst sintering.

Subsequently, $\gamma-\mathrm{Al}_{2} \mathrm{O}_{3}$ was deposited on the pore walls of the membrane via the so-called "urea method" (Gordon et al., 1959). The membrane was first impregnated under vacuum conditions with a solution containing $700 \mathrm{~g} \cdot 1^{-1}$ of $\mathrm{Al}\left(\mathrm{NO}_{3}\right)_{3} \cdot 9 \mathrm{H}_{2} \mathrm{O}$ and $350 \mathrm{gl}^{-1}$ of urea. Afterwards, the impregnated membrane was kept overnight in a closed environment at $95^{\circ} \mathrm{C}$. At these conditions urea decomposes by formation of ammonia whose basicity promotes the precipitation of $\mathrm{Al}(\mathrm{OH})_{3}$ and the subsequent formation of alumina gel. The membrane was then dried at $105^{\circ} \mathrm{C}$ and calcined at $500^{\circ} \mathrm{C}$ for $3 \mathrm{~h}$, enabling $\left(\mathrm{NH}_{4}\right) \mathrm{NO}_{3}$ decomposition, water release from $\mathrm{Al}(\mathrm{OH})_{3}$, and the formation of a transition alumina. 
The calcination temperature was reached at a rate of $2^{\circ} \mathrm{C} \mathrm{min}^{-1}$. The same rate was kept while cooling down to room temperature. The entire procedure was repeated twice, so that the overall content of $\gamma-\mathrm{Al}_{2} \mathrm{O}_{3}$ reached the $4 \%$ mass basis referred to the $\alpha-\mathrm{Al}_{2} \mathrm{O}_{3}$.

The membrane was then Pt-deposited via vacuum impregnation with a $\mathbf{H}_{2} \mathrm{PtCl}_{6}$ aqueous solution followed by drying at $105^{\circ} \mathrm{C}$ overnight and calcination at $500^{\circ} \mathrm{C}$ for $3 \mathrm{~h}$ in calm air. Pt reduction was performed by letting hydrogen permeate the membrane at a slow rate $\left(50 \mathrm{ccmin}^{-1}\right)$ at $400^{\circ} \mathrm{C}$ until $\mathrm{HCl}$ formation ceased. The overall amount of deposited Pt was $1 \%$ mass basis referred to the $\gamma-\mathrm{Al}_{2} \mathrm{O}_{3}$.

\section{MEMBRANE CHARACTERISATION}

\subsection{Destructive analysis}

Some destructive analysis was performed on tube samples to which the same activation route was applied as the membrane carrying ceramic-to-metal sealings at its ends.

BET analysis of the specific surface area of the samples led to a value of $12.5 \mathrm{~m}^{2} \mathrm{~g}^{-1}$, which is consistent with the $220 \mathrm{~m}^{2} \mathrm{~g}^{-1}$, measured by the same means for some $\gamma-\mathrm{Al}_{2} \mathrm{O}_{3}$ powder obtained following the urea method. XRD analysis confirmed that it was actually a transition alumina, while DTA-DTG measurements showed that almost all the water and the $\left(\mathrm{NH}_{4}\right) \mathrm{NO}_{3}$ leave the membrane at lower temperatures than $420^{\circ} \mathrm{C}$.

Figure 5 shows a mercury porosigram of the activated membrane. From these data a mean pore radius of $0.31 \mu \mathrm{m}$ and an overall porosity of $34.6 \%$ were derived. Pt-deposition proved to affect marginally the surface area of the membrane (about $10 \%$ decrease) and negligibly its mean pore radius and porosity.

\subsection{Non-destructive analysis}

For the estimation of $\varepsilon / \tau$ with the above-described technique under reaction conditions, 15 runs were

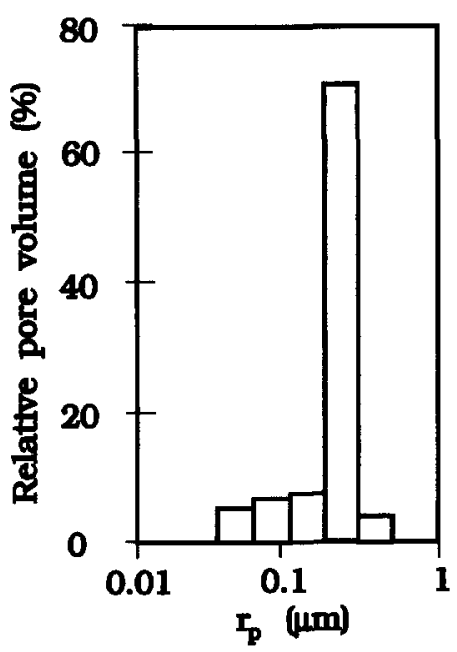

Fig. 5. Hg porosigram of the membrane. performed at different $T_{m}$ values in the range $350-500^{\circ} \mathrm{C}$ feeding a $2 \mathrm{~N} 1 \mathrm{~min}^{-1}$ flow rate of a $4 \%$ propane in $\mathrm{N}_{2}$ mixture at one side of the membrane and an equal flow rate of a $\mathrm{O}_{2} / \mathrm{N}_{2}$ mixture at the opposite side. The $\mathrm{O}_{2}$ content was varied between 4 and $20.95 \%$. Each run was performed feeding the propane either at the shell-side or at the tube-side. In the above ranges of temperature and reactant concentrations no substantial slip of propane and oxygen to their respective opposite sides was observed, and therefore, the existence of a reaction surface was assumed with confidence.

In case external mass transfer resistance is neglected (model D), from the available data and from eq. (4), the effective diffusion coefficients of the reactants could be easily calculated at different temperatures (see Fig. 6). Each data point represents the average value between the estimates derived from shell-feed and tube-feed of propane. By least-squares fitting of the above data, using $\varepsilon / \tau$ as the only fitting parameter the model prediction lines based on eqs (5) and (9) and plotted in the same figure, could be drawn. The bestfit value of $\varepsilon / \tau$ was 0.097 .

When the external mass transfer resistance is accounted for (model $E$ ), the deduction of effective diffusion coefficients from eq. (6) and from the available data is not straightforward. It is nonetheless easy to derive $\varepsilon / \tau$ by fitting the model predictions to the overall conversions measured experimentally. A deviation $\sigma$ can be defined as follows:

$$
\sigma=\frac{\phi_{r}-\left(\phi_{r}\right)_{\mathrm{mod}}}{\phi_{r}}
$$

which is plotted in Fig. 7 for both shell-feed and tube-feed experiments. The optimum $\varepsilon / \tau$ value, derived minimising the sum of all $\sigma^{2}$ values, was in this case 0.124 .

All calculations based on D and E modelling approaches will be performed using their respective, different $\varepsilon / \tau$ estimates. It is easy to explain the difference between the $\varepsilon / \tau$ values obtained from these two models. In both cases the experimental data are satisfactorily fitted, but to achieve this performance, model

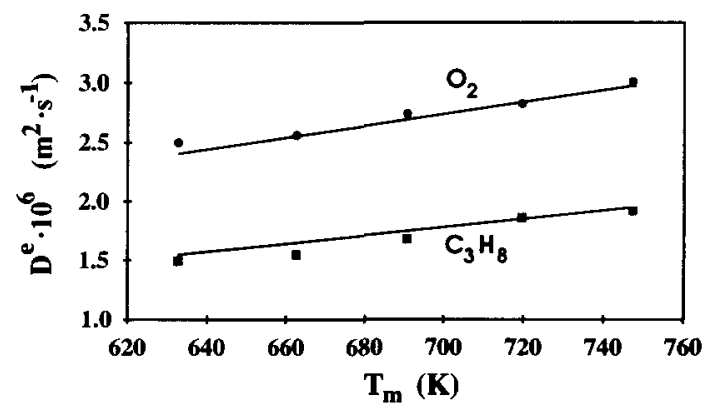

Fig. 6. Effective diffusivities as a function of $T_{m}$ in case external mass transfer is neglected (model D): $(\boldsymbol{\square}),(\bullet)$ data points; (- model D prediction lines for an $\varepsilon / \tau$ best-fit value of 0.097 
$D$, which neglects any external mass transfer resistance, is forced to underestimate $\varepsilon / \tau$, attributing the external resistance to that of the membrane. Therefore, the $\varepsilon / \tau$ prediction, obtained with model $E$, has to be considered as more realistic.

\section{RESULTS OF REACTIVE RUNS}

As underlined above the aim of this study is to describe the performance of the reactor in the transport-controlled regime, where all its interesting features can be exploited. Each reaction run reported hereafter was performed at a shell temperature equal to $490^{\circ} \mathrm{C}$, thus ensuring an almost complete control of mass transport on conversion. Pressure was kept at 2 bar in both feed chambers. The flow rates of the reactant feeds were maintained equal to $2 \mathrm{~N} 1 \mathrm{~min}^{-1}$ each, except during hydrodynamics characterisation runs. After each operating day the membrane was treated overnight with $\mathrm{H}_{2}$ at $350^{\circ} \mathrm{C}$ in order to reconvert to metallic $\mathrm{Pt}$ the eventually formed platinum oxide. At the start of each series of runs the activity of the membrane was checked with a test run (propane concentration $4 \%$, no pressure difference applied). The obtained conversion data were always reproducible within a $3 \%$ deviation.

A set of runs was performed so as to assess the role of the hydrodynamics of the reactant chambers on the performance of the reactor. For different ratios between propane and oxygen feed concentrations $(\omega=0.065-1)$, the residence time of the gas mixtures flowing countercurrently in the two chambers was varied and the corresponding overall conversion was measured. Propane was fed at the tube-side. The flow regime was laminar in all runs $(R e<500)$. In a particular case $(\omega=0.35)$, the flow pattern was changed to cocurrent, and feed sides were inverted (i.e. propane fed at the shell-side). Results are reported in Figs 8 and 9 together with model predictions. Since both models $D$ and $E$ give almost the same results when the correct $\varepsilon / \tau$ values are used only model $E$ lines are reported. Model D predictions based on the more realistic $\varepsilon / \tau$ value of 0.124 are also reported in Fig. 9 so as to appreciate the effect of the external mass transfer resistance on the attainable conversion.

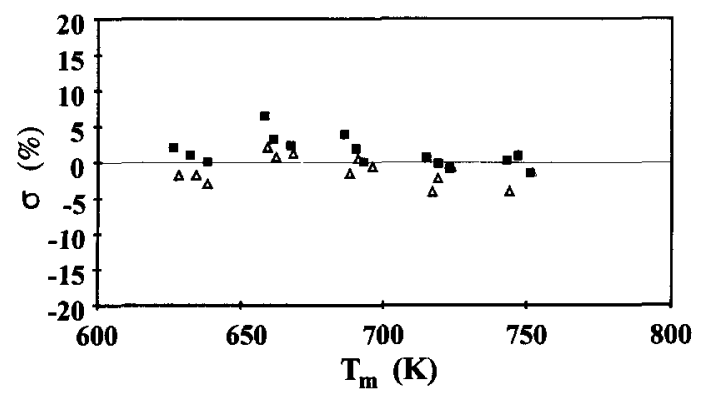

Fig. 7. $\sigma$ as a function of $T_{m}$ (model E). $\varepsilon / \tau$ best-fit value $=0.124$. Propane feed side: $(\square)$ tube; $(\Delta)$ shell.

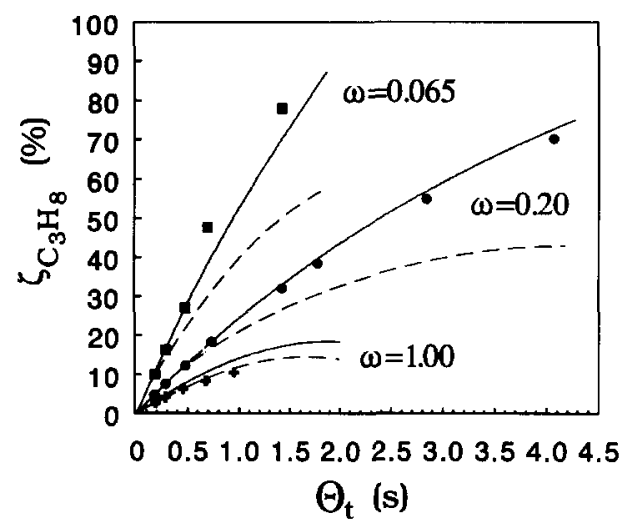

Fig. 8. Accordance of model $\mathbf{E}$ with the experimental conversion data as a function of the residence time $\Theta_{t}$ for different $\omega$ ratios. Experimental data points: $(\mathbf{\square}),(\mathbf{O}),(+)$ model predictions: $(-)$ countercurrent plug-flow; $(-$, CISTR.

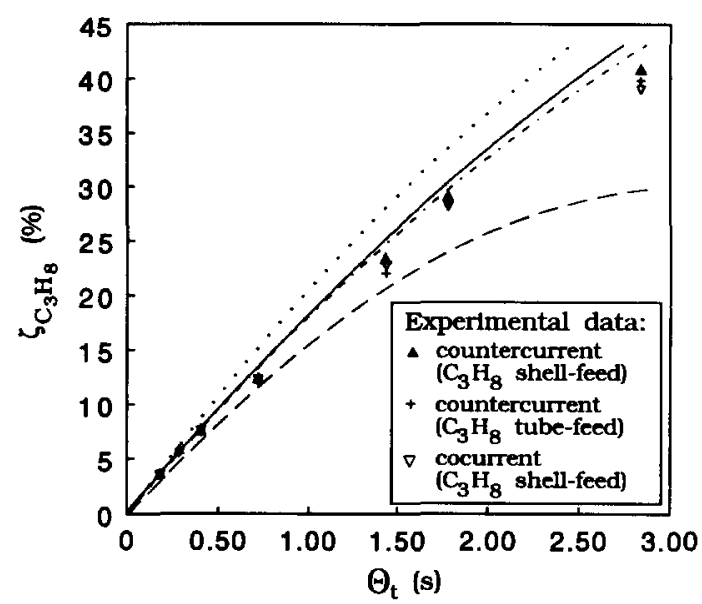

Fig. 9. Accordance of model predictions with experimental conversion data at varying the flow pattern and the propane feed-side for $\omega=0.35$. Model $E$ : ( $\longrightarrow$ ) countercurrent shell-feed; $(--\rightarrow)$ countercurrent tube-feed; $(--)$ cocurrent shell-feed. Model $D(\varepsilon / \tau=0.124):(\cdots \cdots)$ countercurrent tube-feed.

In another series of runs, performed at fixed feed flow rates, the propane concentration was varied from 1 to $100 \%$, meanwhile monitoring the following.

- The variation of the enhancement factor (calculated as the ratio between the measured flow rate of propane entering the membrane under reaction conditions and the maximum physical flow rate at the same operating temperature) for a comparison with the maximum enhancement factor calculated from eq. (8) (Fig. 10). Propane was fed in this case at the tube-side.

- The flow rate of propane entering the membrane and the amount of it which is converted; results 


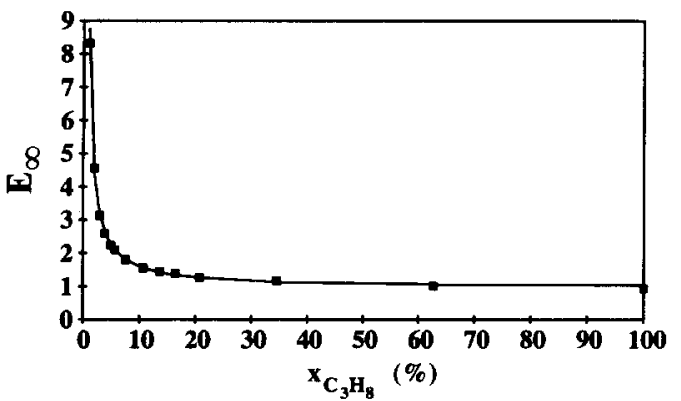

Fig. 10. Variation of the maximum enhancement factor with the propane feed concentration. Comparison between the experimental data ( $\mathbf{\square})$ and model predictions ( $\longrightarrow$ ). Propane feed-side: shell.

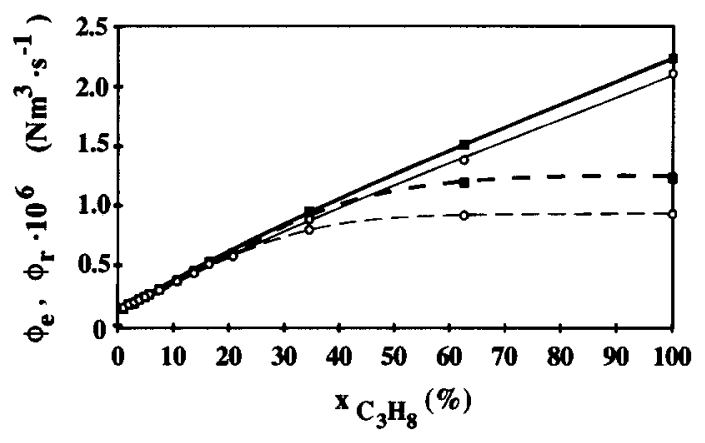

Fig. 11. Comparison between the measured values of $\phi_{e}$ $(\longrightarrow)$ and $\phi_{r}(-\longrightarrow)$ as a function of propane feed concentration for both tube $(O)$ and shell $(\boldsymbol{\omega})$ feed of propane.
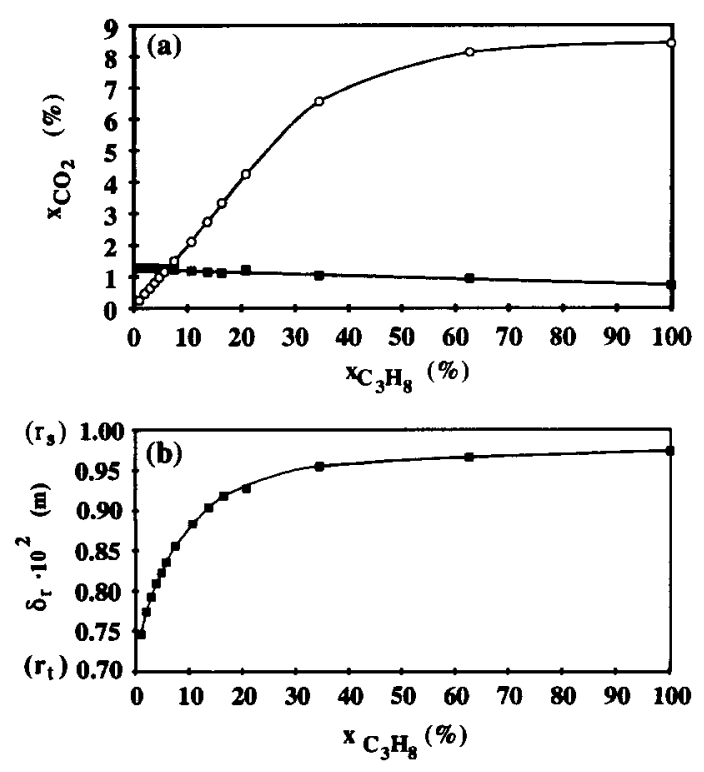

Fig. 12. $\mathrm{CO}_{2}$ distribution at the two membrane sides (a) $[(\square)$ tube side, $(O)$ shell side], and location of the hypothetical reaction surface inside the membrane (b) as a function of the propane feed concentration. Propane feed side: tube. are shown in Fig. 11 for both tube-feed and shell-feed of propane.

- The $\mathrm{CO}_{2}$ distribution at the two membrane sides and the position $\delta_{r}$ of the reaction surface inside the membrane in the above conditions (Fig. 12).

\section{DISCUSSION}

Figure 8 shows that the accordance of model $E$ to the experimental data is satisfactory for a countercurrent plug-flow regime, and that it is generally poor for a CISTR approach. This is quite evident in case reactants are fed in a stoichiometric ratio $(\omega=0.20)$.

In principle, due to a certain temperature difference between the two walls of each annular chamber (e.g. at the tube side common temperatures are ca. $100^{\circ} \mathrm{C}$ for the oil pipe and above $470^{\circ} \mathrm{C}$ for the membrane surface), natural convection might play a role in increasing the degree of mixing of the gas flows. However, Veldsink (1993) calculated that this mechanism can be neglected in the present reactor setup even for such large temperature differences, provided the axial Reynolds number is sufficiently high $(>10)$. Even entry-length effects generating local turbulence can be neglected since the membrane is located sufficiently distant from inlets and outlets. These arguments are consistent with the obtained results, indicating plugflow as a much better assumption than CISTR at least when the reactants are fed in a stoichiometric ratio. Actually, in this case the data points fall just a little below the ideal plug-flow curve. A possible explanation to this may lie in the axial and radial dispersion in the two opposite chambers. Similar observations were made for $\mathrm{CO}$ combustion in the present module (Veldsink, 1993).

When propane is fed in large excess $(\omega=1)$ the measured conversions fall slightly below the model curves either based on a plug-flow or a CISTR regime. This may be explained as follows. As reported in Paper II of this series (Saracco et al., 1995) a radial asymmetry of the catalytic activity of the membrane was detected. Particularly, the tube side of the membrane appeared to be more active than the shell-side of it. When high propane concentrations are fed at the tube side of the membrane, the reaction zone shifts towards the shell-side of the membrane, where the catalytic activity is lower. Kinetics may be too low to convert the entire flux of propane that enters the membrane, thus allowing a certain slip of propane in the shell chamber. A small slip of propane was actually noticed during these runs $\left(\mathrm{S}_{\mathrm{C}_{3} \mathrm{H}_{8}}<3 \%\right)$. Obviously, both model schemes, which are based on the simplifying assumption of considering the reaction confined in a cylindrical surface inside the membrane, tend to overestimate the attainable conversion.

When propane is fed at low concentrations (below the stoichiometric value) a couple of measured conversion values are surprisingly higher than those predicted by the plug-flow model. This is a likely consequence of the uncertainties affecting the various measurements (leading to the above quoted $15 \%$ maximum absolute deviation in the verification of 
overall mass balances) and to some phenomena which cannot be accounted for by such measurements (for example, existence of localised temperature variations within and along the membrane which cannot be measured by the single thermocouple).

From data plotted in Fig. 9, it is once again clear that the plug-flow assumption is in any case better than the CISTR one. The conversions attained feeding propane at the shell-side are slightly higher than those measured when feeding propane at the opposite side. This can also be explained with the presence of a catalytic activity profile inside the membrane. Particularly, when propane is fed at the shell-side its stoichiometric excess "pushes" the reaction zone towards the more active tube-side of the membrane, thus enabling higher conversions than with a tubeside feed of propane.

As concerns the comparison between the cocurrent and the countercurrent flow patterns, this last one gives slightly higher conversions, as predicted also by the model. Further considerations to this merit can be drawn from Fig. 13, where the position of the reaction surface [eq. (7)] was plotted as a function of the axial coordinate of the membrane for both flow patterns, for given $\omega$ and $\Theta_{t}$ values. Despite the fact that the cocurrent operation mode gives lower conversions than the countercurrent one, the former might be preferred whenever the avoidance of any slip of reactant becomes a primary goal. The cocurrent flow pattern allows to keep the reaction zone more inside the membrane along its entire length despite the excess of propane. In this case the bulk concentrations of the reactants inside their respective chambers decrease simultaneously along the same direction. Conversely, during countercurrent feed runs slip of reactants becomes more critical, especially at the extremes of the reactor where the membrane mediates the contact between a fresh inlet gas (high reactant concentration) and an outlet gas mixture whose reactant concentration has been depleted to some extent by reaction. If one of the reactants is fed in large stoichiometric excess and the residence time in the module is rather high, the situation will become particularly critical at one end of the membrane.

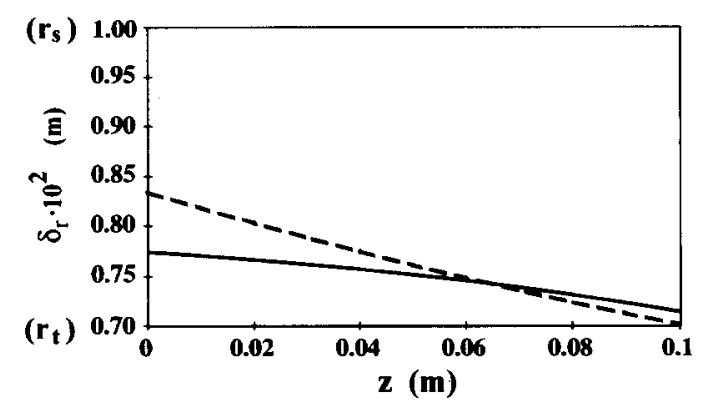

Fig. 13. Location of the hypothetical reaction surface along the membrane length as predicted by model $E$ for either cocurrent $(-)$ or countercurrent $(--)$ flow of reactants and for tube feed of propane. $\omega=0.35 ; \Theta_{t}=4 \mathrm{~s}$.
A further issue concerns the effect of the external mass transfer in affecting the overall attainable conversion. By comparing the dotted and the dashedand-dotted lines in Fig. 9 it can be deduced that this effect is limited: the external mass transfer resistance entails approximately a $10 \%$ reduction of the maximum conversion attainable.

The simplified model based on the plug-flow assumption gives particularly accurate estimates of the experimentally measured maximum enhancement factor as a function of propane concentration (Fig. 10). This is particularly encouraging for those data obtained at low propane concentration, when the presence of reaction in the membrane markedly enhances the flux of the key reactant entering the membrane, $\mathrm{N}_{\mathrm{C}_{3} \mathrm{H}_{8}}\left(r_{t}\right)$, compared with non-reactive conditions. When the propane concentration is relatively high $(>20 \%$ ), the presence of reaction only marginally influences $\mathrm{N}_{\mathrm{C}_{3} \mathrm{H}_{3}}\left(r_{t}\right)$, and the enhancement factor remains close to unity. In this case it is much more relevant to establish if the membrane is capable of converting the entire flux of propane getting into it, despite the remarkable stoichiometric mismatch of the concentrations of propane and oxygen. Figure 11 elucidates this point. When propane is fed at the shell-side any slip of propane is prevented for concentrations lower than $35 \%$. Beyond this limit, the conversion is still increased but at much lower rate while more and more important slip of propane is detected at the air-side. Similar behaviour is obtained when propane is fed at the tube-side, but in this case slip becomes noticeable already with a $20 \%$ propane concentration, and the maximum attainable concentration is lower. Once again the explanation to this difference is easily deduced from the lower catalytic activity of the shell-side of the membrane where the reaction zone is pushed at increasing the concentration of propane, when this one is fed at the tube-side. This is clearly represented in Fig. 12 showing the $\mathrm{CO}_{2}$ distribution at the two membrane sides and the consequent migration of the reaction zone within the membrane.

The maximum attainable conversion in the presence or in the absence of slip, among others, depends on the structural parameters of the membrane (e.g. pore diameter, porosity, tortuosity, amount and distribution of the catalytic phase added). The obtained results enlighten some interesting features of this reactor concept, although they do not represent the optimal performance attainable with it. Further, in the next paper of this series (Saracco et al., 1995), the application of a pressure difference over the membrane will be demonstrated to be a powerful tool to increase the overall conversion.

\section{CONCLUSIONS}

A membrane reactor with separate feed of reactants was applied to the catalytic combustion of propane with air. A number of interesting properties is enabled when operating the reactor in the transport-controlled regime: absence of slip (the reaction takes place 
entirely inside the membrane), easy controllability (flow rates, composition and pressure of each flow rate can be varied independently), thermal runaways are hampered (transport is much less temperature sensitive than kinetics), etc. (Veldsink et al., 1992).

The behaviour of the reactor, when no pressure differences are applied across the membrane and diffusive transport of reactants controls the attainable conversion, was investigated. Wide operation ranges have been found in which the reaction in the membrane is self-sustaining (no additional heat supply is necessary) and the reactor properly functions as a heat generator. The hydrodynamics of the two chambers separated by the catalytic membrane were demonstrated to be close to plug-flow behaviour. The obtained propane conversions are moreover in good agreement with an isothermal modelling approach, solved analytically, which proved to be sufficiently accurate to describe the performance of the reactor under the above operating conditions.

It has to be once again underlined that the structural parameters of the membrane may dramatically influence the performance of the reactor (e.g. membrane thickness, trans-membrane pore size, amount and distribution of catalytic phase added). In the present study the pore size was chosen in the so-called transition region between the Knudsen and the Poiseuille mass transfer regimes. This ensured a high mass transfer resistance in the membrane compared to that in the gas phase outside of it. In fact, the reduction of the attainable conversion ascribable to the effect of the external mass transfer resistance was esteemed to be lower than about $10 \%$ of the maximum attainable conversion for $\omega$ equal to 0.35 . The membrane thickness was chosen so as to have reasonable mechanical resistance, the amount of catalyst in order to have a sufficient catalytic activity.

The proposed isothermal modelling should allow to assess which are the best structural parameters to be used so as to attain the optimum performance of this reactor setup when no pressure differences are applied over the membrane and ultimately its economic feasibility compared with alternative technologies.

In Paper II (Saracco et al., 1995) it will be shown how the application of a pressure difference over the membrane can be regarded as an effective mean to increase the per pass conversion in the reactor, without losing, within certain limits, its most peculiar properties.

Acknowledgement - The authors acknowledge the financial support of Gastech (Apeldoorn, The Netherlands).

\section{NOTATION}

$D$ diffusion coefficient, $\mathrm{m}^{2} \mathrm{~s}^{-1}$

$E$ enhancement factor

$\Delta H^{\circ} \quad$ heat of reaction in standard conditions, $\mathrm{J} \mathrm{mol}^{-1}$

$k_{g} \quad$ mass transfer coefficient, $\mathrm{m} \mathrm{s}^{-1}$

$\begin{array}{ll}K_{0} & \text { Knudsen constant, } \mathrm{m} \\ K_{1}, K_{2} & \text { accessory parameters } \\ L & \text { membrane thickness, } \mathrm{m} \\ M & \text { molecular mass, } \mathrm{kg} \mathrm{mol}^{-1} \\ N & \text { mole flux, mol } \mathrm{m}^{-2} \mathrm{~s}^{-1} \\ p & \text { partial pressure, } \mathrm{Pa} \\ P & \text { absolute pressure, } \mathrm{Pa} \\ r & \text { radius, } \mathrm{m} \\ R & \text { universal gas constant }\left(=8.314 \mathrm{~J} \mathrm{~mol}^{-1}\right. \\ & \mathrm{K}-1) \\ R e & \text { Reynolds number } \\ S & \text { slip fraction } \\ T & \text { temperature, } \mathrm{K} \\ \langle T\rangle & \text { mixing-cup temperature, } \mathrm{K} \\ v & \text { diffusion volume } \\ x & \text { mole fraction } \\ \langle x\rangle & \text { mixing-cup mole fraction } \\ z & \text { axial coordinate }\end{array}$

\section{Greek letters}

$\alpha$

$\delta_{r} \quad$ location of hypothetical reaction surface inside the membrane, $\mathrm{m}$

porosity

per-pass conversion throughout the module

$\Theta \quad$ residence time, $\mathrm{s}$

$v \quad$ stoichiometric coefficient

$\sigma \quad$ deviation parameter defined in eq. (15)

$\tau \quad$ tortuosity

$\phi \quad$ volumetric flow rate, $\mathrm{N} \mathrm{m}^{3} \mathrm{~s}^{-1}$

$\phi_{e} \quad$ volumetric flow rate of propane entering

the membrane, $\mathrm{N} \mathrm{m}^{3} \mathrm{~s}^{-1}$

$\phi_{r} \quad$ volumetric flow rate of propane converted, $\mathrm{N} \mathrm{m}^{3} \mathrm{~s}^{-1}$

$\omega$

ratio between the inlet flow rates of propane and oxygen

Subscripts

$A, B, C, i, j$ generic components

in, out inlet, outlet

$m$ membrane

mod model prediction

oil oil pipe

$p \quad$ pore

ph physical, non-reactive conditions

$s, t \quad$ shell-, tube-side of the membrane

shell shell of the reactor module

$\infty \quad$ infinitely fast reaction

\section{Superscripts}

$\begin{array}{ll}e & \text { effective } \\ 0 & \text { gas phase }\end{array}$

\section{REFERENCES}

Gordon, L., Salutsky, M. L. and Willard, H. H., 1959, Precipitation from Homogeneous Solutions. Chapman \& Hall, New York. 
Lundberg, R. E., McCuen, P. A. and Reynolds, W. C., 1963 , Heat transfer in annular passages. Hydrodynamically developed laminar flow with arbitrarily prescribed wall temperatures or heat fluxes. Int. J. Heat Mass Transfer 6 , 459-529.

Reid, R. C., Prausnitz, J. M. and Poling, B. E., 1987, The Properties of Gases and Liquids, IV Edition. McGraw-Hill, New York.

Saracco, G. and Specchia, V., 1994, Catalytic inorganic membrane reactors: present experience and future opportunities. Catal. Rev. - Sci. Engng 36, 305-384.

Saracco, G., Veldsink, J. W., Versteeg, G. and van Swaaij, W. P. M., 1995, Catalytic combustion of propane in a membrane reactor with separate feed of reactants-II. Operation in presence of trans-membrane pressure gradients. Chem. Engng Sci. (in press).

Saracco, G., Versteeg, G. and van Swaaij, W. P. M., 1994, Current hurdles to the success of high-temperature membrane reactors. J. Membrane Sci. 95, 105-123.

Sloot, H. J., 1991, A non-permselective membrane reactor for catalytic gas phase reactions. Ph.D. dissertation, University of Twente, The Netherlands.
Sloot, H. J., Smolders, C. A., van Swaaij, W. P. M. and Versteeg, G. F., 1992, High-temperature membrane reactor for catalytic gas-solid reactions. A.I.Ch.E. J., 38, 887-900.

Sloot, H. J., Versteeg, G. F. and van Swaaij, W. P. M., 1990, A non-permselective membrane reactor for chemical processes normally requiring strict stoichiometric feed of reactants. Chem. Engng Sci. 45, 2415-2421.

Tsotsis, T. T., Champagnie, A. M., Minet, R. G. and Liu, P. K. T., 1993, Catalytic membrane reactors, in Computer-aided Design of Catalysts (Edited by Becker and Pereira). Marcel Dekker, New York.

Veldsink, J. W., 1993, A catalytically active, non-permselective membrane reactor for kinetically fast, strongly exothermic heterogeneous reactions. Ph.D. dissertation, University of Twente, The Netherlands.

Veldsink, J. W., van Damme, R. M. J., Versteeg, G. F. and van Swaaij, W. P. M., 1992, A catalytically active membrane reactor for fast, heterogeneously catalysed reactions. Chem. Engng Sci. 47, 2939-2944.

Westerterp, K. R., van Swaaij, W. P. M. and Beenakers, A. A. C. M., 1983, Chemical Reactor Design and Operation, 2nd Edition. Wiley, New York. 